\title{
A Low-Complexity Resource Allocation for Multiple Access Passive IoT System
}

\author{
Shiying Han ${ }^{1, *}$ and Zixiong Wang ${ }^{2}$ \\ 1 College of Electronic Information and Optical Engineering, Nankai University, Tianjin 300350, China \\ 2 School of Electrical and Information Engineering, Tianjin University, Tianjin 300072, China; \\ wangzixiong@tju.edu.cn \\ * Correspondence: syhan@nankai.edu.cn
}

Received: 6 November 2019; Accepted: 26 November 2019; Published: 28 November 2019

\begin{abstract}
An ambient backscatter communication (AmBC) system with multiple backscatter devices (BDs) is investigated in this work. The cooperative reader receives the information from the primary transmitter (PT) and the multiple BDs simultaneously. With the asymptotic signal-to-noise-plus-interference ratio (SINR) of the BDs, an optimization problem that jointly optimizes the reflection coefficients of BDs and the primary transmit power is formulated. Considering that the adaptive optimization of reflection coefficients according to the instantaneous primary channel state information (CSI) is unaffordable in practice, we propose a low-complexity resource allocation scheme, which results in a long-term configuration of the BD reflection coefficients before the primary transmit power is allocated. With the long-term reflection coefficients, the transmit power of the primary system is optimized by solving the transformed two cascaded optimization problems which have closed-form solutions. Simulation results are provided to demonstrate the effectiveness of the proposed scheme.
\end{abstract}

Keywords: Internet-of-Things; ambient backscatter communications; multiple access; resource allocation; symbiotic radio

\section{Introduction}

Future wireless networks will be integrated with machine type communication (MTC) to provide various Internet-of-Things (IoT) services. It has been foreseen that billions of IoT devices will be deployed worldwide by 2020 [1]. Different from the ultra-reliable and low-latency communication (uRLLC), the massive MTC (mMTC) aims to accommodate massive connections of IoT devices that are featured by small size, simple hardware, and limited power supply. Although short package is normally transmitted by each IoT device, it still poses big challenge for the management of radio spectrum and power resource [2]. To deal with this challenge, the ambient backscatter communication $(\mathrm{AmBC})$ technique has been proposed to enable the transmission of passive IoT device without extra spectrum and energy cost [3-6], which has been recognized as a promising technique to prolong the lifetime of IoT device and pave the way to the sustainable IoT system.

In the AmBC system, the passive IoT device, also known as backscatter device (BD), transmits its data by backscattering the incident ambient RF signal via intentionally changing its impedance of antenna. The RF signal can be a TV, cellular, or WiFi signal that carries information. From the perspective of spectrum sharing, the BD concurrently shares the primary spectrum while sharing the RF source. However, different from the traditional concurrent spectrum sharing where the transmission of secondary system is always harmful to the primary system, the BD transmission here can be constructive or destructive to the primary system. When the bit rate of $\mathrm{BD}$ is equal or comparable to that of the primary signal, the BD signal is an interference to the primary system, and thus the AmBC 
system is "parasitic" to the primary system. In contrary, when the BD data rate is far lower than the primary bit rate, the backscatter signal can be treated as a useful multipath if proper channel estimation is available. In this case, the AmBC system is "commensal" with the primary system. Therefore, from the perspective of spectrum sharing, the coexistence between the AmBC system and the primary system is also known as symbiotic radio (SR), which can operates at the parasitic mode or commensal mode [7].

The existing literatures on the AmBC and SR systems can be mainly classified into two categories: One category focuses on the detection of $\mathrm{BD}$ signal, whereas the other category focuses on the resource allocation and optimization for the SR system. Without channel state information (CSI) in the SR system, the authors of $[8,9]$ propose signal detection schemes with the aid of differential coding and Manchester coding at the BD, respectively. Seeing that the direct link signal is a strong interference at the $\mathrm{BD}$ reader, the authors of $[10,11]$ design the BD waveform so that the signal can be detected from the cyclic prefix and the guard band of the OFDM symbol, respectively. In [12], a label-assisted learning approach is proposed for detecting the BD signal, and in [13], the multi-antenna technique is employed to cancel the strong in-band interference. To get the CSI of the SR system, the authors of $[14,15]$ propose the channel estimation scheme using single-antenna and multiple-antenna reader, respectively. By assuming that the perfect CSI is known, the transmit power of primary system and the reflection coefficient of the BD are jointly optimized in [16,17].

Although the SR system with single BD has been widely investigated in the literature, there still exists a lack of study on the multi-BD SR system, which is more practical for the mMTC scenario. For example, the central processor needs to collect information from multiple BDs, which are distributed within a geographical region. In [18], an SR system with multiple BDs is investigated, where the full-duplex access point (AP) transmits its primary signal to the mobile station while receiving the backscattered signal from the BDs. Time-division multiple access (TDMA) is adopted as the multiple access scheme of the BDs. The transmit power of AP, reflection coefficient, and access time for each $\mathrm{BD}$ are jointly optimized. In [19], the nonorthogonal multiple access (NOMA) is adopted to accommodate the multiple BDs, which are differentiated by the different reflection coefficients. Note that the existing multiple access schemes proposed in the literature highly depend on the coordination among BDs. For example, in the TDMA scheme, each BD needs to know when it should start its transmission and how long it can transmit. In the NOMA scheme, on the other hand, the reflection coefficients should be carefully designed so that the multiple BDs can be differentiated. Thus, each reflection coefficient should be transmitted to its intended $\mathrm{BD}$, which is challenging in practice. To avoid the coordination among BDs, we propose a random code based multiple access scheme in [20]. It has been shown that when the reflection coefficients and primary transmit power are jointly optimized, the optimal reflection coefficients are closely related to the instantaneous CSI of the primary system. This means that the optimal reflection coefficients should be configured as frequently as the channel of primary system varies, which is unaffordable in practice for the passive IoT devices.

In the joint optimization problem, the reflection coefficients and the primary transmit power are coupled by the constraint of primary rate requirement. To solve this problem, in this work, we decouple the optimization of the reflection coefficients and the primary transmit power. The long-term reflection coefficients are first derived, which are independent on the instantaneous CSI of the primary system, but only dependent on the distance-based path loss for each link and the systematic parameters, such as the BD system load (defined as the ratio of the number of BDs to the number of subcarriers) and the transmit power budget of the primary system. With the long-term reflection coefficients, we optimize the primary transmit power according to the primary instantaneous CSI to achieve its target rate and maximize the $\mathrm{BD}$ achievable rate. To further decrease the complexity of algorithm, we transform the power allocation problem into two cascaded optimization problems which have closed-form solution. By adopting the proposed scheme, frequent configuration of reflection coefficients can be avoided and the overall complexity of the resource allocation for the SR system is reduced. Simulation results are provided to demonstrate the effectiveness of the proposed scheme. 
The remainder of the paper is organized as follows. The system model is presented in Section 2, based on which the signal transmission is analyzed. Then the joint resource allocation problem is formulated and solved in Section 3. The simulation results are given in Section 4. Finally, the conclusion is drawn in Section 5.

\section{System Model}

We consider a multi-BD AmBC system as shown in Figure 1. The reader receives the signals from the primary transmitter (PT) and the K BDs simultaneously. The primary system adopts orthogonal frequency division multiplexing (OFDM) with $N$ subcarriers. Each transmission link is comprised by distance-based path loss, shadowing, and small-scale fading, where the shadowing is treated to be one for simplicity. Flat-fading is assumed for each subcarrier and the channel condition is unchanged within the channel covariance time.

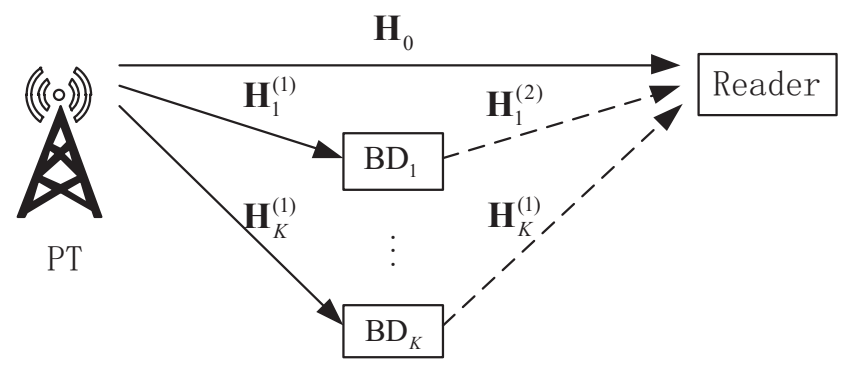

Figure 1. An ambient backscatter communication (AmBC) system with $K$ backscatter devices (BDs) and a cooperative receiver/reader which receives the information from the primary transmitter (PT) and from the BDs simultaneously.

The transmission link directly from the PT to the reader is referred to as direct link, and that from the PT to the reader via each BD is referred to as backscatter link. We denote $\mathbf{H}_{0}=\operatorname{diag}\left(H_{0,1}, \ldots, H_{0, N}\right)$ as the channel response matrix of the direct link, where $H_{0, n}$ is the channel response of subcarrier $n$. Accordingly, the channel gain of subcarrier $n$ is denoted by $g_{0, n}$. Similarly, $\mathbf{H}_{k}^{(i)}=\operatorname{diag}\left(H_{k, 1}^{(i)}, \ldots, H_{k, N}^{(i)}\right)$ denotes the channel response matrix for the backscatter link via $\mathrm{BD} k$, where $i=1$ represents the link from PT to BD $k$ and $i=2$ represents the link from BD $k$ to the reader. Assuming $\mathbf{H}_{k}^{(1)}$ and $\mathbf{H}_{k}^{(2)}$ are independent with each other, the compound channel response matrix of backscatter link via $\mathrm{BD}$ $k$ can be written as $\mathbf{H}_{k}=\mathbf{H}_{k}^{(1)} \mathbf{H}_{k}^{(2)}$. Accordingly, the channel gains for these links are denoted by $\left\{g_{k, n}^{(1)}\right\}_{n=1, \ldots, N},\left\{g_{k, n}^{(2)}\right\}_{n=1, \ldots, N}$ and $\left\{g_{k, n}\right\}_{n=1, \ldots, N}$, respectively.

Let vector $\mathbf{p}=\left[p_{1}, \ldots, p_{N}\right]$ denote the primary transmit power, where $p_{n}$ is the transmit power on subcarrier $n$. The transmission signal from the PT can be represented as $\mathbf{s}=\left[s_{1}, \ldots, s_{N}\right]$, where $s_{n}$ 's are independent with each other and $\mathrm{E}\left[\left|s_{n}\right|^{2}\right]=p_{n}$. CP is inserted to take care of the intersymbol interference. Let $\mathbf{W}$ be the $N \times N$ Fourier transform matrix. The baseband received signal at $\mathrm{BD}, k$, and at the reader via direct link can be, respectively, written as

$$
\begin{aligned}
\mathbf{x}_{k} & =\mathbf{W}^{H} \mathbf{H}_{k}^{(1)} \mathbf{s}, \\
\mathbf{y}_{\mathrm{d}} & =\mathbf{W}^{H} \mathbf{H}_{0} \mathbf{s} .
\end{aligned}
$$

$\mathrm{BD} k$ backscatters $\mathbf{x}_{k}$ by intentionally changing the antenna impedance according to a random code with length of $N$. Let $\tilde{\mathbf{c}}_{k}=\left[c_{k, 1}, \ldots, c_{k, N}\right]^{T}$ be the random code which is independently chosen by $\mathrm{BD} k$. It has $\mathrm{E}\left[\left|c_{k, n}\right|^{2}\right]=1$ and $\mathrm{E}\left[c_{i, n} c_{j, n}^{*}\right]=0$ for $i \neq j$. Thus, it can be seen that the random code is a sequence of +1 and -1 . When $c_{k, n}=1$, the $n$-th bit of OFDM symbol, i.e., $x_{k}(n)$, is unchanged; while when $c_{k, n}=-1$, the phase of $x_{k}(n)$ is changed by $\pi$. Thus, the backscattered signal from $\mathrm{BD} k$ can be written as $\mathbf{x}_{k}^{\prime}=\mathbf{W}^{H} \mathbf{H}_{k}^{(1)} \mathbf{S} \tilde{\mathbf{c}}_{k} \tilde{a}_{k}$, where $\tilde{a}_{k}$ is the data of BD $k, \mathbb{E}\left[\left|\tilde{a}_{k}\right|^{2}\right]=\tilde{\alpha}_{k} \in[0,1]$ and 
$\mathbf{S}=\operatorname{diag}\left(s_{1}, \ldots, s_{N}\right)$. For the convenience of expression, we denote $\mathbf{c}_{k}=\frac{1}{\sqrt{N}} \tilde{\mathbf{c}}_{k}$ and $a_{k}=\sqrt{N} \tilde{a}_{k}$. Then, $\mathbf{x}_{k}^{\prime}$ can be rewritten as

$$
\mathbf{x}_{k}^{\prime}=\mathbf{W}^{H} \mathbf{H}_{k}^{(1)} \mathbf{S c}_{k} a_{k}
$$

where $\mathbb{E}\left[\left|a_{k}\right|^{2}\right]=N \tilde{\alpha}_{k} \triangleq \alpha_{k}$ and $\mathbb{E}\left[\mathbf{c}_{k} \mathbf{c}_{k}^{H}\right]=\frac{1}{N} \mathbf{I}$. For simplicity, both $\tilde{\alpha}_{k}$ and $\alpha_{k}$ are referred to as the reflection coefficient of $\mathrm{BD} k$, although $\alpha_{k}$ is $N$ times of $\tilde{\alpha}_{k}$. Then, the received signal at the reader via backscatter links can be written as

$$
\mathbf{y}_{\mathbf{b}}=\sum_{i=1}^{K} \mathbf{W}^{H} \mathbf{H}_{i} \mathbf{S c}_{i} a_{i}
$$

Thus, the compound baseband received signal at the reader is

$$
\mathbf{y}=\mathbf{y}_{\mathrm{d}}+\mathbf{y}_{\mathrm{b}}+\mathbf{n},
$$

where $\mathbf{n}$ is the additive white Gaussian noise (AWGN) with zero mean and variance $\sigma^{2}$.

At the reader receiver, the $\mathrm{CP}$ is removed from $\mathbf{y}$, which then passes through the fast Fourier transform (FFT) processor, yielding the output

$$
\tilde{\mathbf{y}}=\mathbf{H}_{0} \mathbf{s}+\sum_{i=1}^{K} \mathbf{H}_{i} \mathbf{S} \mathbf{c}_{i} a_{i}+\tilde{\mathbf{n}},
$$

where $\tilde{\mathbf{y}}=\mathbf{W y}$ and $\tilde{\mathbf{n}}=\mathbf{W n}$. We consider a large-dimensional system where the numbers of the BDs and the subcarriers go to infinity, i.e., $K, N \rightarrow \infty$. The ratio between $K$ and $N$ approaches a constant, i.e., $\frac{K}{N} \rightarrow \beta$, where the constant $\beta$ is defined as the BD system load. Moreover, rich multipath is assumed, and the wireless channel follows uniform power delay profile [21]. Note that the strength of the direct link signal $\mathbf{y}_{\mathbf{d}}$ is much stronger than that of the backscatter-link signal $\mathbf{y}_{\mathbf{b}}$, due to the two-hop path loss and reflection loss. Thus, the reader can firstly detect the primary signal by regarding the backscatter component as interference. Therefore, the signal-to-noise-plus-interference ratio (SINR) of the primary signal on the $n$-th subcarrier can be derived as

$$
\gamma_{0, n}=\frac{p_{n} g_{0, n}}{\frac{1}{N} \sum_{i=1}^{K} g_{i, n} \alpha_{i} p_{n}+\sigma^{2}}, n=1, \ldots, N
$$

Given that $g_{i, n}$ is comprised by distance-based path loss, denoted by $l_{i}$, and small-scale fading, denoted by $\tilde{g}_{i, n}$, with unit mean, $K \rightarrow \infty, \gamma_{0, n}$ can be further derived as

$$
\gamma_{0, n}=\frac{p_{n} g_{0, n}}{\frac{1}{N} \sum_{i=1}^{K} l_{i} \alpha_{i} p_{n}+\sigma^{2}} .
$$

We consider a uniform level of $l_{i} \alpha_{i}$ for all $i=1, \ldots, K$. Thus, the asymptotic SINRs of all BDs are the same at the reader. Then, $\gamma_{0, n}$ can be expressed as

$$
\gamma_{0, n}=\frac{p_{n} g_{0, n}}{\beta \bar{\alpha} p_{n}+\sigma^{2}},
$$

where $\bar{\alpha}=l_{i} \alpha_{i}, \forall i$. Equation (9) indicates that the SINR of the primary system on each subcarrier is related to the $\mathrm{BD}$ system load. A larger amount of BDs introduces higher interference to the primary system. Moreover, the SINR of the primary signal is also related to the BD reflection coefficient observed at the receiver side, which is referred to as the received BD reflection coefficient for short. It shows that a higher received BD reflection coefficient leads to a lower primary SINR, as higher interference is introduced by the backscatter transmission. 
When the primary signal is detected, the direct-link component, i.e., $\mathbf{H}_{0} \mathbf{s}$, can be removed from the received signal by using successive interference cancellation (SIC). Suppose that the SIC is perfect and there is no residual interference, the remained signal is

$$
\sum_{i=1}^{K} \mathbf{H}_{i} \mathbf{S c}_{i} a_{i}+\tilde{\mathbf{n}}
$$

Different from the traditional code-division multiple access (CDMA) system where the user data is only modulated by the spreading code and channel [22], here the BD data is also modulated by the power allocation matrix of the primary system. When the match-filter (MF) receiver is adopted at the reader, the asymptotic SINR for each $\mathrm{BD}$ is given by [20]

$$
\gamma(\bar{\alpha}, \mathbf{p})=\frac{\left(\frac{1}{N} \sum_{n=1}^{N} p_{n}\right)^{2} \bar{\alpha}}{\frac{1}{N} \sum_{n=1}^{N} p_{n}^{2} \beta \bar{\alpha}+\frac{\sigma^{2}}{N} \sum_{n=1}^{N} p_{n}} .
$$

When a minimum mean square error (MMSE) receiver is adopted, the asymptotic SINR for each BD satisfies the function

$$
\gamma(\bar{\alpha}, \mathbf{p})=\frac{\frac{1}{N} \sum_{n=1}^{N} p_{n} \bar{\alpha}}{\frac{1}{N} \sum_{n=1}^{N} p_{n} \frac{\beta \bar{\alpha}}{1+\gamma(\bar{\alpha}, \mathbf{p})}+\sigma^{2}}
$$

\section{Low-Complexity Reflection Coefficient Configuration and Power Allocation}

\subsection{Problem Formulation}

The joint optimization of the BD reflection coefficients and the primary power allocation can be formulated as

$$
\begin{aligned}
& \underline{\mathrm{P} 1}: \max _{\alpha_{k} \in[0, N], \mathbf{p} \geq 0} \gamma(\bar{\alpha}, \mathbf{p}) \\
& \text { s.t. } \quad \frac{2}{N} \sum_{n=1}^{N} \log _{2}\left(1+\gamma_{0, n}\right) \geq R_{0} \\
& w_{k} \geq w_{0}, \forall k \\
& \sum_{n=1}^{N} p_{n} \leq P_{\max } .
\end{aligned}
$$

The objective of $\mathrm{P} 1$ is to maximize the asymptotic BD SINR and thereby to maximize the BD rate. Equation (13) is the constraint of primary rate, where $R_{0}$ denotes the target rate of the primary transmission. The factor $\frac{2}{N}$ is added for highlighting the spectral efficiency achieved by the primary transmission. Equation (14) indicates that the harvested power at each BD, denoted by $w_{k}$, should be enough to cover the power consumption, denoted by $w_{0}$, which is used for switch changing among antenna impedances and driving the micro processor at the BD. Letting $\eta$ be the energy harvesting efficiency, $w_{k}$ can be derived as $\eta\left(1-\frac{\alpha_{k}}{N}\right) \sum_{n=1}^{N} p_{n} g_{k, n}^{(1)}$. Consider that $p_{n}$ is allocated independently with $g_{k, n}^{(1)}$, and as $N \rightarrow \infty, \frac{1}{N} \sum_{n=1}^{N} g_{k, n}^{(1)} \rightarrow \frac{1}{N} \sum_{n=1}^{N} \tilde{g}_{k, n}^{(1)} l_{k}^{(1)}=l_{k}^{(1)}$, where $l_{k}^{(1)}$ is the path loss in $g_{k, n}^{(1)}$. Thus, $w_{k}$ can be further derived as $\eta\left(1-\frac{\alpha_{k}}{N}\right) l_{k}^{(1)} \sum_{n=1}^{N} p_{n}$. Equation (15) is the total power constraint, where $P_{\max }$ denotes the power budget of the PT.

Sorting (14), we have $\sum_{n=1}^{N} p_{n} \geq \max _{k}\left\{\frac{w_{0}}{\eta\left(1-\frac{\alpha_{k}}{N}\right) l_{k}^{(1)}}\right\}$. Jointly considering (15), we can see that P1 can be feasible only when $\max _{k}\left\{\frac{w_{0}}{\eta\left(1-\frac{\alpha_{k}}{N}\right) l_{k}^{(1)}}\right\} \leq P_{\max }$. As we consider a uniform level of $l_{i} \alpha_{i}$ for all $i=1, \ldots, K$ and have denoted $\bar{\alpha}=l_{i} \alpha_{i}$, P1 can be transformed as the joint optimization of $\bar{\alpha}$ and $\mathbf{p}$. Let $\mathcal{S}_{\bar{\alpha}}$ be the feasible region of $\bar{\alpha}$. Given $\alpha_{k} \in[0, N], \mathcal{S}_{\bar{\alpha}}$ can be derived as $\mathcal{S}_{\bar{\alpha}}=\left[0, \bar{\alpha}_{\text {max, },}\right]$, 
where $\bar{\alpha}_{\text {max }, 1}=\min _{k}\left\{l_{k} N\left(1-\frac{w_{0}}{\eta P_{\max } l_{k}^{(1)}}\right)\right\}$. Then, (14) always holds provided $\bar{\alpha} \in \mathcal{S}_{\bar{\alpha}}$. Thus, (14) can be omitted and $\mathrm{P} 1$ can be recast as

$$
\underline{\mathrm{P} 2}: \max _{\bar{\alpha} \in \mathcal{S}_{\bar{\alpha}}, \mathbf{p} \succeq 0} \gamma(\bar{\alpha}, \mathbf{p}) \text {, s.t. (13), (15). }
$$

\subsection{Long-Term Reflection Coefficient}

Based on the objective function of P2 and the constraint (13), we can see that the optimal $\bar{\alpha}$ is closely related to the instantaneous CSI of the primary system. This means that if the BD reflection coefficients are configured according to the optimal $\bar{\alpha}$, which is derived from P2, it requires frequent configuration of $\mathrm{BD}$ reflection coefficients, and, consequently, frequent signaling between the primary system and the BD system is needed. To tackle this problem, we propose to find the long-term reflection coefficient which is independent with the primary instantaneous CSI. This can be achieved before the primary transmit power is allocated. Although the long-term reflection coefficient for each BD is suboptimal, it can avoid frequent configuration and reduce the intersystem signaling. To solve the long-term reflection coefficients from $\mathrm{P} 2$, the following proposition is given.

Proposition 1. Let $\bar{p}$ and $\sigma_{p}^{2}$ denote $\frac{1}{N} \sum_{n=1}^{N} p_{n}$ and $\frac{1}{N} \sum_{n=1}^{N} p_{n}^{2}-\left(\frac{1}{N} \sum_{n=1}^{N} p_{n}\right)^{2}$, respectively. The asymptotic $B D$ SINR is increasing over $\bar{p}$ while decreasing over $\sigma_{p}^{2}$.

Proof. For MF receiver, Equation (11) can be rewritten as

$$
\gamma(\bar{\alpha}, \mathbf{p})=\frac{\bar{p}^{2} \bar{\alpha}}{\left(\sigma_{p}^{2}-\bar{p}^{2}\right) \beta \bar{\alpha}+\sigma^{2} \bar{p}}=\frac{\bar{\alpha}}{\left(\frac{\sigma_{p}^{2}}{\bar{p}^{2}}-1\right) \beta \bar{\alpha}+\frac{\sigma^{2}}{\bar{p}}},
$$

based on which we can observe that $\gamma(\bar{\alpha}, \mathbf{p})$ is increasing over $\bar{p}$ while decreasing over $\sigma_{p}^{2}$.

For MMSE receiver, denoting $\gamma(\bar{\alpha}, \mathbf{p})$ by $x$, Equation (12) can be rewritten as

$$
x=\frac{\bar{p} \bar{\alpha}}{\bar{p} \frac{\beta \bar{\alpha}}{1+x}+\sigma^{2}}
$$

which can be further sorted as

$$
\frac{\bar{\alpha}}{x}-\frac{\beta \bar{\alpha}}{1+x}=\frac{\sigma^{2}}{\bar{p}}
$$

The right-hand side of Equation (18) is decreasing over $\bar{p}$. The left-hand side of Equation (18) is an decreasing function over $x$ given $\beta \leq 1$. Thus, $x$ is increasing as $\bar{p}$ increases. Moreover, it should be noted that $\gamma(\bar{\alpha}, \mathbf{p})$ in Equation (12) is an upper-bound approximation of the real asymptotic BD SINR [22]. Its tightness can be improved by minimizing the variance of transmit power across subcarriers, i.e., $\sigma_{p}^{2}$. Thus, given $\bar{p}$ and thereby $\gamma(\bar{\alpha}, \mathbf{p})$, the real asymptotic BD SINR will approach $\gamma(\bar{\alpha}, \mathbf{p})$ as $\sigma_{p}^{2}$ decreases. In other words, although $\gamma(\bar{\alpha}, \mathbf{p})$ is independent with $\sigma_{p}^{2}$, the real asymptotic BD SINR is decreasing over $\sigma_{p}^{2}$. Thus, the proposition is proved.

Proposition 1 tells that the optimal power allocation should meet $\sum_{n=1}^{N} p_{n}=P_{\max }$ to maximize the BD SINR. Moreover, the power variance $\sigma_{p}^{2}$ should be minimized. Therefore, given the BD reflection coefficient, the primary transmit power can be allocated according to

$$
\min _{\mathbf{p}} \sum_{n=1}^{N} p_{n}^{2}, \quad \text { s.t., } \sum_{n=1}^{N} p_{n}=P_{\max }
$$


whose solution follows equal power allocation, i.e., $p_{n}=\frac{P_{\max }}{N}=p$, for all $n=1, \ldots, N$. With this power allocation strategy, the primary achievable rate can be derived as

$$
R_{p}=\frac{2}{N} \sum_{n=1}^{N} \log _{2}\left(1+\frac{p g_{0, n}}{\beta \bar{\alpha} p+\sigma^{2}}\right)(\mathrm{bps} / \mathrm{Hz})
$$

As $N \rightarrow \infty, R_{p}$ approaches $2 \mathbb{E}\left[\log _{2}\left(1+\frac{p g_{0, n}}{\beta \bar{\alpha} p+\sigma^{2}}\right)\right]$, where the expectation is taken over $\left\{g_{0, n}\right\}$. Considering that $g_{0, n}$ follows exponential distribution with unit mean, $R_{p}$ can be further derived as

$$
R_{p}=-\frac{2}{\ln 2} e^{A} \operatorname{Ei}(-A)
$$

where $\operatorname{Ei}(x)=\int_{-\infty}^{x} \frac{e^{t}}{t} d t$ and $A=\beta \bar{\alpha}+\frac{\sigma^{2}}{p}$. Then, we can obtain the long-term reflection coefficient by solving

$$
\underline{\mathrm{P} 2.1}: \max _{\bar{\alpha} \in \mathcal{S}_{\bar{\alpha}}} \gamma(\bar{\alpha}, p), \text { s.t. }-\frac{2}{\ln 2} e^{A} \operatorname{Ei}(-A) \geq R_{0} .
$$

Note that $\gamma(\bar{\alpha}, p)$ is monotonically increasing over $\bar{\alpha}$, while $-\frac{2}{\ln 2} e^{A} \operatorname{Ei}(-A)$ is monotonically decreasing over $\bar{\alpha}$. Thus, we can have that the solution of P2.1 is

$$
\bar{\alpha}^{*}=\min \left\{\bar{\alpha}_{\max , 1}, \bar{\alpha}_{\max , 2}\right\},
$$

where $\bar{\alpha}_{\text {max }, 1}$ is the maximum feasible value of $\bar{\alpha}$ in $\mathcal{S}_{\bar{\alpha}}$, and $\bar{\alpha}_{\max , 2}$ is the solution of $-\frac{2}{\ln 2} e^{A} \operatorname{Ei}(-A)=$ $R_{0}$. As $-\frac{2}{\ln 2} e^{A} \operatorname{Ei}(-A)$ is a decreasing function over $\bar{\alpha}$, the solution of $-\frac{2}{\ln 2} e^{A} \operatorname{Ei}(-A)=R_{0}$ can be obtained by using bisection search efficiently. With $\bar{\alpha}^{*}$, the reflection coefficient for BD $k$ can be derived as $\alpha_{k}=\bar{\alpha}^{*} / l_{k}$ for each $k=1, \ldots, K$. Note that $\bar{\alpha}^{*}$ is independent with the instantaneous CSI for both the primary system and the BD system, and thus no frequent reconfiguration of the BD reflection coefficient is needed.

\subsection{Low-Complexity Primary Power Allocation}

Given $\bar{\alpha}=\bar{\alpha}^{*}$ and the instantaneous CSI of the primary system, i.e., $\left\{g_{0, n}\right\}$, the power allocation problem of the PT can be formulated as

$$
\begin{aligned}
\underline{\mathrm{P} 2.2}: \max _{\mathbf{p} \succeq 0} \gamma\left(\bar{\alpha}^{*}, \mathbf{p}\right) \\
\text { s.t. } \frac{2}{N} \sum_{n=1}^{N} \log _{2}\left(1+\frac{p_{n} g_{0, n}}{\beta \bar{\alpha}^{*} p_{n}+\sigma^{2}}\right) \geq R_{0} .
\end{aligned}
$$

Based on Proposition 1, P2.2 can be equivalently transformed as

$$
\underline{\mathrm{P} 2.2^{\prime}}: \min _{\mathbf{p} \succeq 0} \sum_{n=1}^{N} p_{n}^{2}, \quad \text { s.t. }(24), \quad \sum_{n=1}^{N} p_{n}=P_{\max } \text {. }
$$

It can be observed that the equal power allocation is optimal if constraint (24) is satisfied with $p_{n}=\frac{P_{\max }}{N}$. Otherwise, the transmit power should be reallocated to fulfill the primary target rate. It can be seen that P2.2' is a convex optimization problem, and thus can be solved by dual decomposition 
method [23]. Let $\lambda_{1} \geq 0$ and $\lambda_{2} \geq 0$ be the dual variables associated with (24) and (25), respectively. By using dual decomposition, the $n$-th partial Lagrange function can be written as

$$
L_{p}\left(p_{n}, \lambda_{1}, \lambda_{2}\right)=p_{n}^{2}-\lambda_{1} \log _{2}\left(1+\frac{p_{n} g_{0, n}}{p_{n} \beta \bar{\alpha}^{*}+\sigma^{2}}\right)-\lambda_{2} p_{n}
$$

Then, the optimal power allocation $p_{n}^{*}$ can be derived by solving $\frac{\partial L_{p}}{\partial p_{n}}=0$.

It can be seen that the closed-form $p_{n}^{*}$ is hard to obtain through solving $\frac{\partial L_{p}}{\partial p_{n}}=0$. To reduce the complexity introduced by numerically solving $\frac{\partial L_{p}}{\partial p_{n}}=0$ in each iteration, we transform P2.2' into the following cascaded problems,

$$
\begin{aligned}
& \underline{\text { P3.1 }}: \min _{\mathbf{0} \succeq 0} \sum_{n=1}^{N} o_{n}, \text { s.t. } \sum_{n=1}^{N} \log _{2}\left(1+\frac{o_{n} g_{0, n}}{\beta \bar{\alpha}^{*} o_{n}+\sigma^{2}}\right)=R_{0}, \\
& \underline{\text { P3.2 }}: \min _{\mathbf{q} \succeq 0} \sum_{n=1}^{N}\left(q_{n}+o_{n}^{*}\right)^{2}, \text { s.t. } \sum_{n=1}^{N}\left(q_{n}+o_{n}^{*}\right)=P_{\max },
\end{aligned}
$$

where $\mathbf{o}=\left[o_{1}, \ldots, o_{N}\right]$ and $\mathbf{q}=\left[q_{1}, \ldots, q_{N}\right]$ are the power allocation vectors for the first-stage and the second-stage power allocation, respectively. P3.1 aims to minimize the total transmit power required to meet the primary target rate. P3.2 aims to allocate the remained power to minimize the power variance across subcarriers. It can be seen that both of the problems can be readily solved via dual decomposition method. Thus, their closed-form solutions can be derived as

$$
\begin{aligned}
& o_{n}^{*}=\left(-\frac{1}{2}\left(\frac{\sigma^{2}}{\beta \bar{\alpha}^{*}}+\frac{\sigma^{2}}{\beta \bar{\alpha}^{*}+g_{0, n}}\right)+\sqrt{\left.\frac{1}{4}\left(\frac{\sigma^{2}}{\beta \bar{\alpha}^{*}}+\frac{\sigma^{2}}{\beta \bar{\alpha}^{*}+g_{0, n}}\right)^{2}+\frac{\kappa g_{0, n} \sigma^{2}-\sigma^{4}}{\beta \bar{\alpha}^{*}\left(\beta \bar{\alpha}^{*}+g_{0, n}\right)}\right)^{+},}\right. \\
& q_{n}^{*}=\left(\frac{\delta}{2}-o_{n}^{*}\right)^{+},
\end{aligned}
$$

where $\kappa$ and $\delta$ are the non-negative dual variables that make the constraints of P3.1 and P3.2 hold, respectively. Then, the optimal power allocation follows $p_{n}^{*}=o_{n}^{*}+q_{n}^{*}$ for each $n=1, \ldots, N$.

Based on the above analysis, the overall low-complexity algorithm to solve P1 can be summarized as in Algorithm 1.

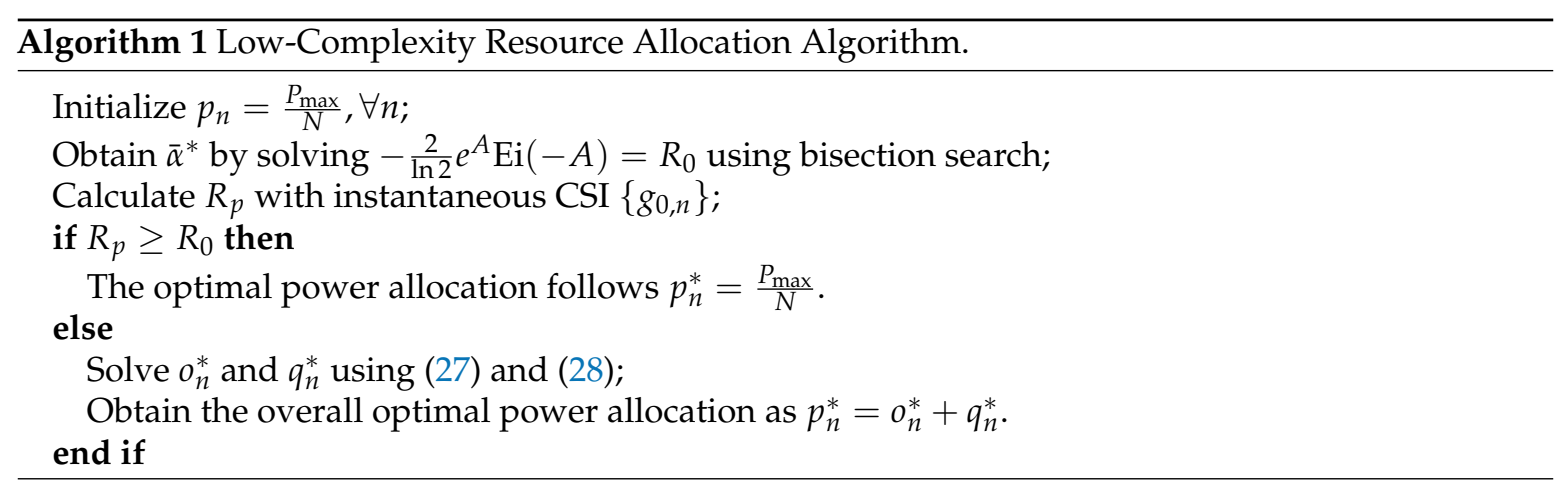

\section{Simulation Results}

In the simulation, we set the number of subcarriers $N$ as 256 and the number of multipath as $N / 8$. The transmit power budget $P_{\max }$ is set as $25 \mathrm{~dB}$. Without loss of generality, the distance-based path loss for each link is normalized as one; thus, the uniformed received reflection coefficient $\bar{\alpha}$ can reflect the reflection coefficient $\alpha_{k}, \forall k$. Moreover, we assume a small value of $\frac{w_{0}}{\eta P_{\max }}$ so that the feasible region of $\bar{\alpha}$ is $\mathcal{S}_{\bar{\alpha}}=[0, N]$. We illustrate the performance when the primary rate constraint (24) cannot be met with 
$p_{n}=\frac{P_{\max }}{N}$. As benchmarks, we also simulate the performance when water-filling power allocation (WFPA) is adopted to solve P2.2' and the performance with the adaptive optimization of reflection coefficient according to the primary instantaneous CSI [20].

We first observe the performance of the AmBC system by varying BD system load $\beta$ with $R_{0}=0.8$. Figure 2 illustrates the achievable asymptotic BD SINR. It can bee seen that for both MF and MMSE receivers, the BD SINR with our proposed scheme is lower than that with the adaptive optimization scheme, but higher than that with the WFPA scheme. Note that the gap between performance with the adaptive optimization and that with the proposed scheme is negligible small when MF receiver is adopted and as the BD system load (i.e., the number of BDs) increases. For the MMSE receiver, the performance gap between the proposed scheme and the adaptive optimization scheme also decreases as $\beta$ increases. This indicates that by using the proposed scheme, the complexity of the resource allocation for multi-BD SR system can be profoundly reduced with small sacrifice of BD SINR, especially for the MF case.

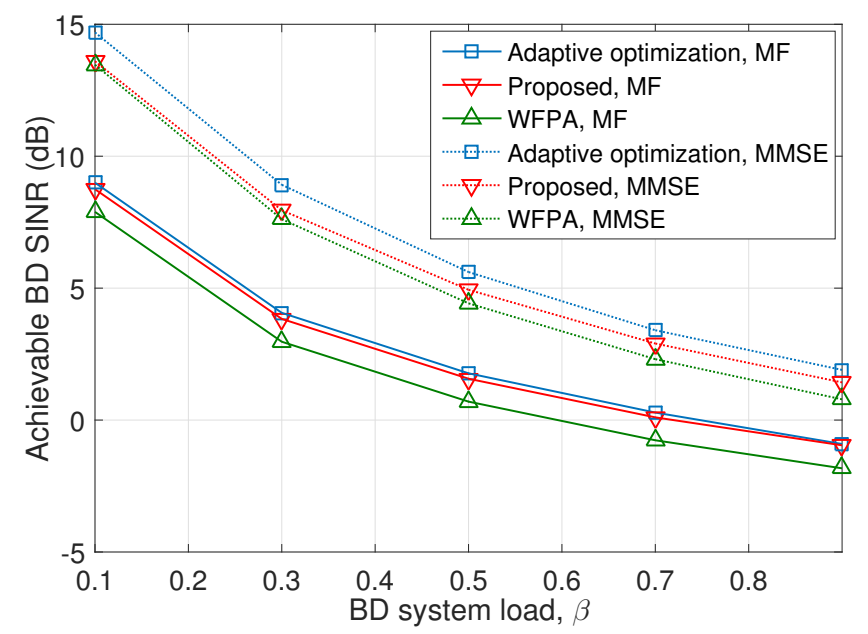

Figure 2. Achievable asymptotic BD signal-to-noise-plus-interference ratio (SINR) versus BD system load.

Figure 3 illustrates the reflection coefficient $\frac{\alpha^{*}}{N}$ under different levels of $\beta$. It indicates that for both MF and MMSE receivers, the reflection coefficient obtained by the proposed algorithm is lower than that achieved by the adaptive optimization. The gap is more obvious when the BD system load is small. This means that, compared with the adaptive optimization of reflection coefficient, although a degree of BD SINR is sacrificed, a smaller portion of reflection energy is needed with the proposed scheme. Thus, more energy of the incident signal can be reserved for prolonging the lifetime of the device or used for receiving signal to realize the tag writing [24]. On the other hand, we can see that the $\mathrm{BD}$ reflection coefficient achieved with the proposed scheme is equal to that achieved by the WFPA. This indicates that the proposed scheme can achieve higher BD data rate than the WFPA scheme by using the same level of reflection coefficient.

Figure 4 illustrates the achievable primary rate with different BD system loads. We can see that all the three schemes can guarantee the primary target rate, i.e., $0.8 \mathrm{bps} / \mathrm{Hz}$. However, the primary rates achieved with the proposed scheme and the WFPA scheme are both higher than that achieved with the adaptive optimization scheme. This indicates that the spectrum sharing potential is not fully utilized with the proposed scheme. Compared with WFPA; however, the primary rate achieved by the proposed scheme is lower than that achieved by the WFPA, meaning that the proposed algorithm can explore the spectrum sharing opportunity better than the WFPA scheme.

Finally, we observe the achievable BD data rate by varying the target rate of the primary transmission to illustrate the impact of the primary target rate on the AmBC system. Different numbers of BDs are considered, i.e., $K=25$ and 75 , to represent the scenarios of light and heavy BD 
system load, respectively. From Figure 5, we can see that for both MF and MMSE cases, and for all levels of feasible $R_{0}$, the proposed scheme can achieve higher BD data rate than the WFPA scheme. The gap is more obvious when MF is adopted. Specifically, for the MF receiver, the decreasing speed of the $\mathrm{BD}$ rate as the primary target rate increases is relatively small when $R_{0}$ is less than $1.5 \mathrm{bps} / \mathrm{Hz}$. In contrast, for the MMSE receiver, the BD data rate can be increased quickly when the primary target rate decreases, especially with the larger number of BDs $(K=75)$.

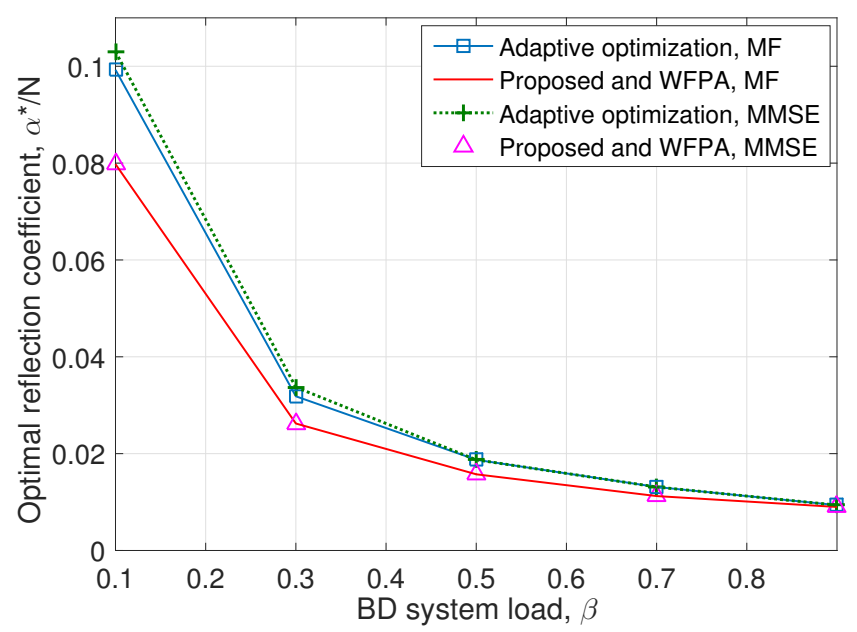

Figure 3. BD reflection coefficient versus BD system load.

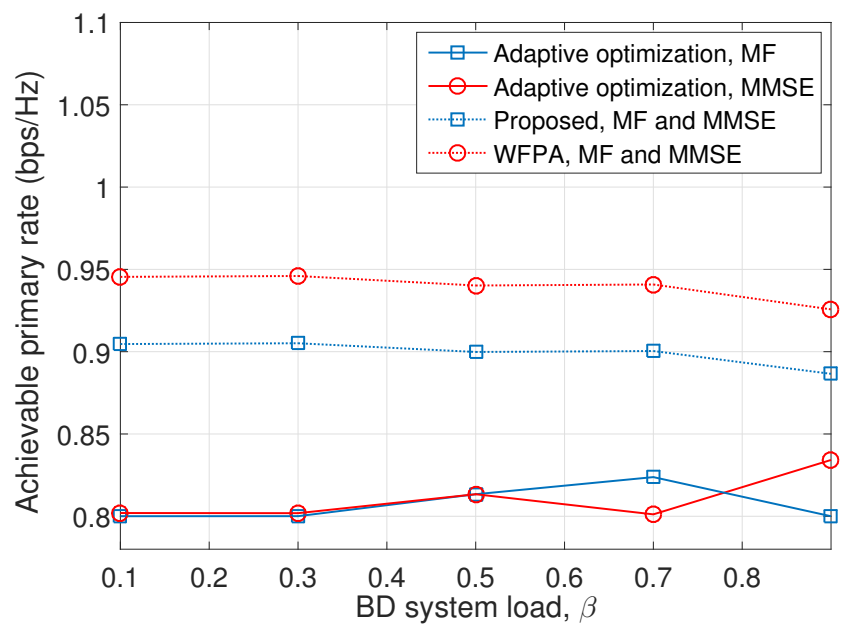

Figure 4. Achievable primary rate versus BD system load. 


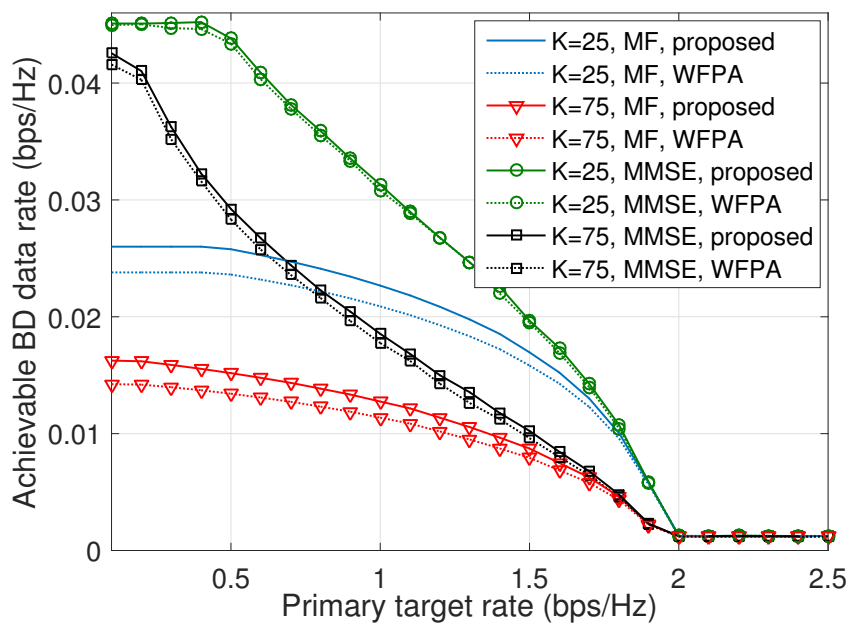

Figure 5. The achievable BD data rate versus primary target rate.

\section{Conclusions}

In this paper, the $\mathrm{BD}$ reflection coefficient configuration and the primary power allocation have been investigated for a multi-BD SR system. To release the dependency of the reflection coefficient configuration with the instantaneous CSI, we decoupled the joint optimization of reflection coefficients and the primary power allocation by finding the long-term reflection coefficients. With the long-term BD reflection coefficients, we reduce the complexity of primary power allocation by transforming the power allocation problem into two cascaded optimization problems, both of which are with closed-form solutions. Simulation results have demonstrated that the proposed scheme can achieve good comprehensive performance in terms of BD achievable rate and BD reflection coefficient with low-complexity algorithm.

Author Contributions: S.H. modeled the system as well as formulating and solving the problem. Z.W. ran the simulation to verify the proposed scheme and algorithm. S.H. and Z.W. wrote and revised the manuscript, respectively.

Funding: This research was funded by the National Natural Science Foundation of China (NSFC, grant number: 61601321, 61601247) and the Natural Science Foundation of Tianjin City (grant number: 17JCQNJC01500).

Conflicts of Interest: The authors declare no conflicts of interest.

\section{References}

1. European Union, Identification and Quantification of Key Socio-Economic Data to Support Strategic Planning for the Introduction of 5G in Europe-SMART 2014/0008; European Union: Luxembourg, 2016.

2. Zhang, L.; Liang, Y.; Xiao, M. Spectrum sharing for internet of things: A survey. IEEE Wireless Commun. 2019, 26, 132-139. [CrossRef]

3. Liu, V.; Parks, A.; Talla, V.; Gollakota, S.; Wetherall, D.; Smith, J.R. Ambient backscatter: Wireless communication out of thin air. In Proceedings of the SIGCOMM '13 Proceedings of the ACM SIGCOMM 2013 conference on SIGCOMM, Hong Kong, China, 12-16 August 2013; pp. 39-50.

4. Hoang, D.T.; Niyato, D.; Wang, P.; Kim, D.I.; Han, Z. Ambient backscatter: A new approach to improve network performance for RF powered cognitive radio networks. IEEE Trans. Commun. 2017, 65, 3659-3674. [CrossRef]

5. Van Huynh, N.; Hoang, D.T.; Lu, X.; Niyato, D.; Wang, P.; Kim, D.I. Ambient backscatter communications: A contemporary survey. IEEE Commun. Surv. Tutor. 2018, 20, 2889-2922. [CrossRef]

6. Liang, Y.-C.; Long, R.; Zhang, Q.; Chen, J.; Cheng, H.V.; Guo, H. Large intelligent surface/antennas (LISA): Making reflective radios smart, J. Commun. Inf. Netw. 2019, 4, 40-50.

7. Guo, H.; Liang, Y.; Long, R.; Zhang, Q. Cooperative ambient backscatter system: A symbiotic radio paradigm for passive IoT. IEEE Wirel. Commun. Lett. 2019, 8, 1191-1194. [CrossRef] 
8. Wang, G.; Gao, F.; Fan, R.; Tellambura, C. Ambient backscatter communication systems: Detection and performance analysis. IEEE Trans. Commun. 2016, 64, 4836-4846. [CrossRef]

9. Tao, Q.; Zhong, C.; Lin, H.; Zhang, Z. Symbol detection of ambient backscatter systems with Manchester coding. IEEE Trans. Wirel. Commun. 2018, 17, 4028-4038. [CrossRef]

10. Yang, G.; Liang, Y.; Zhang R.; Pei, Y. Modulation in the air: Backscatter communication over ambient OFDM carrier. IEEE Trans. Commun. 2018, 66, 1219-1233. [CrossRef]

11. ElMossallamy, M.A.; Pan, M.; Jäntti, R.; Seddik, K.G.; Li, G.Y.; Han, Z. Noncoherent backscatter communications over ambient OFDM signals. IEEE Trans. Commun. 2019, 67, 3597-3611. [CrossRef]

12. Zhang, Q.; Guo, H.; Liang, Y.; Yuan, X. Constellation learning-based signal detection for ambient backscatter communication systems. IEEE J. Sel. Areas Commun. 2019, 37, 452-463. [CrossRef]

13. Guo, H.; Zhang, Q.; Xiao S.; Liang, Y. Exploiting multiple antennas for cognitive ambient backscatter communication. IEEE Internet Things J. 2019, 6, 765-775. [CrossRef]

14. Ma, S.; Wang, G.; Fan, R.; Tellambura, C. Blind channel estimation for ambient backscatter communication systems. IEEE Commun. Lett. 2018, 22, 1296-1299. [CrossRef]

15. Zhao, W.; Wang, G.; Atapattu, S.; He, R.; Liang, Y. Channel estimation for ambient backscatter communication systems with massive-antenna reader. IEEE Trans. Vehi. Technol. 2019, 68, 8254-8258. [CrossRef]

16. Kang, X.; Liang, Y.; Yang, J. Riding on the primary: A new spectrum sharing paradigm for wireless-powered IoT devices. IEEE Trans. Wirel. Commun. 2018, 17, 6335-6347. [CrossRef]

17. Guo, H.; Liang, Y.; Long, R.; Xiao, S.; Zhang, Q. Resource allocation for symbiotic radio system with fading channels. IEEE Access 2019, 7, 34333-34347. [CrossRef]

18. Yang, G.; Zhang, Q.; Liang, Y. Cooperative ambient backscatter communications for green internet-of-things. IEEE Internet Things J. 2018, 5, 1116-1130. [CrossRef]

19. Guo, J.; Zhou, X.; Durrani, S.; Yanikomeroglu, H. Design of nonorthogonal multiple access enhanced backscatter communication. IEEE Trans. Wirel. Commun. 2018, 17, 6837-6852. [CrossRef]

20. Han, S.; Liang, Y.; Chen, Q.; Wang, Z.; Sun, G. The design and optimization of multiple access symbiotic radio system. IEEE Internet Things J. 2019, under review.

21. Goldsmith, A. Wireless Communications; Cambridge University Press: Cambridge, UK, 2005.

22. Han, S.; Liang, Y.; Soong, B. Spectrum refarming: A new paradigm of spectrum sharing for cellular networks. IEEE Trans. Commun. 2015, 63, 1895-1906. [CrossRef]

23. Boyd, S.; Vandenberghe, L. Convex Optimization; Cambridge University Press: Cambridge, UK, 2004.

24. Long, R.; Guo, H.; Zhang, L.; Liang, Y. Full-duplex backscatter communications in symbiotic radio systems. IEEE Access 2019, 7, 21597-21608. [CrossRef]

(C) 2019 by the authors. Licensee MDPI, Basel, Switzerland. This article is an open access article distributed under the terms and conditions of the Creative Commons Attribution (CC BY) license (http://creativecommons.org/licenses/by/4.0/). 